\title{
The influence of an infectious disease on a prey-predator model equipped with a fractional-order derivative
}

\author{
Salih Djilali ${ }^{1,2}$ and Behzad Ghanbari ${ }^{3,4^{*}}$ (1)
}

\section{"Correspondence:}

b.ghanbary@yahoo.com

${ }^{3}$ Department of Basic Science,

Kermanshah University of

Technology, Kermanshah, Iran

${ }^{4}$ Department of Mathematics,

Faculty of Engineering and Natural

Sciences, Bahçeşehir University,

34349 Istanbul, Turkey

Full list of author information is

available at the end of the article

\begin{abstract}
In this research, we discuss the influence of an infectious disease in the evolution of ecological species. A computational predator-prey model of fractional order is considered. Also, we assume that there is a non-fatal infectious disease developed in the prey population. Indeed, it is considered that the predators have a cooperative hunting. This situation occurs when a pair or group of animals coordinate their activities as part of their hunting behavior in order to improve their chances of making a kill and feeding. In this model, we then shift the role of standard derivatives to fractional-order derivatives to take advantage of the valuable benefits of this class of derivatives. Moreover, the stability of equilibrium points is studied. The influence of this infection measured by the transmission rate on the evolution of predator-prey interaction is determined. Many scenarios are obtained, which implies the richness of the suggested model and the importance of this study. The graphical representation of the mathematical results is provided through a precise numerical scheme. This technique enables us to approximate other related models including fractional-derivative operators with high accuracy and efficiency.
\end{abstract}

Keywords: Predator-prey model; Infected prey; Hunting cooperation; Fractional calculus

\section{Highlights}

- A fractional-order predator-prey system with prey infection is investigated.

- The equilibrium points of the system are determined and the necessary conditions for their existence and stability are analyzed.

- An efficient numerical method is employed to determine system behaviors.

- Several graphical representations are presented for the explanation of the mathematical results.

\section{Introduction}

Infectious diseases arise when contaminated foreign bodies enter the human body. These foreign bodies are germs, viruses, fungi, or parasites. These bodies are transmitted through infection from another human being, animals, contaminated food, or from exposure to any of the environmental factors that are contaminated with any of these bodies.

C) The Author(s) 2021. This article is licensed under a Creative Commons Attribution 4.0 International License, which permits use, sharing, adaptation, distribution and reproduction in any medium or format, as long as you give appropriate credit to the original author(s) and the source, provide a link to the Creative Commons licence, and indicate if changes were made. The images or other third party material in this article are included in the article's Creative Commons licence, unless indicated otherwise in a credit line to the material. If material is not included in the article's Creative Commons licence and your intended use is not permitted by statutory regulation or exceeds the permitted use, you will need to obtain permission directly from the copyright holder. To view a copy of this licence, visit http://creativecommons.org/licenses/by/4.0/. 
These infections have many symptoms on the body, including high body temperature and pain, in addition to other symptoms that differ according to the site of infection, type, and severity of the infection. It is possible to have an infection that causes mild symptoms, and therefore it does not need to be treated. On the other hand, there are serious cases that may cause death. In some cases, they may affect the population balance of many species in the environment. In a worse scenario, some species may even become extinct due to some deadly diseases developed in some highly sensible populations. Mathematical models for predicting the evolution of species have been used in an increasing way in the last few decades. It started from Lotka and Volterra models [27, 37], where their usefulness in avoiding many worst scenarios for numerous species as extinction was proved. Nowadays scientists use this tool for revealing the outcome of a certain strategy taken by some governments in dealing with some species, which can be considered as an important tool for conserving all the species existing nowadays.

The ecological species are most sensitive to any infection that appears, which can influence the evolution of some species, as an epitome. We consider a predator-prey interaction. This infection can affect the strength of some predators and the efficiency of hunting, which puts some predators in danger of extinction. In the literature, many works have investigated the predator-prey interaction in the presence of infectious diseases. For instance, please refer to the papers $[9,25,26,41]$. On the other hand, there are many strategies that the predators can consider for achieving a successful hunt. One of the most effective strategies is the predator hunting cooperation, where many predators gather to hunt a single prey. This method is very useful in reducing the hunting fail rate. Many hunters behave in this way. For example, lions, hyenas, and wild dogs are known for the highefficiency rate in hunting. The mathematical modeling of this specific behavior of predator was modeled for the first time in [22], where a simple model was used for describing such a cooperation. Until now, there have been a few works that study such a behavior in the predator-prey interaction, we mention as an example the works of [8, 10, 29-33, 38, 39]. Hence, it is worth investigating the influence of an infectious disease in the predator-prey interaction in the presence of predator hunting cooperation, which is going to be the principal subject of this research. We consider a three-species model that considers an infection developed in a prey population (which means that the prey population is divided into two species: the susceptible prey and the infected prey), and because of the opportunism of the predator, we can consider that the predator hunts the two types of prey. Based on the obtained results in [40], it is obtained that the time-fractional derivative has extensive applications in describing many real-life situations, which is known by memory effect of a dynamical system; memory rate is called for the order of the derivative, and the memory function for the kernel of the factional derivative. The time-fractional derivative is used for modeling many real-world phenomena, we mention as an example [20, 23, 24]. So its role is indispensable in the mathematical modeling of some ecological-epidemiological behaviors. To get more models of prey and hunter-modeled under the influence of a disease, we encourage interested readers to refer to the relevant literature in $[7,9,10,13-21,23-$ $25,29,31,34-36,39,41]$. Some other general problems can be found in $[1-6,28]$. 
Following the above-mentioned trends, we consider the following eco-epidemiological model:

$$
\left\{\begin{array}{l}
\mathscr{D}^{\alpha} S(t)=r(S+I)-(\lambda+a P) P S-\delta S I-\mu S, \\
\mathscr{D}^{\alpha} I(t)=\delta S I-(\lambda+a P) P I-\mu I, \\
\mathscr{D}^{\alpha} P(t)=e(\lambda+a P) P(S+I)-m P,
\end{array}\right.
$$

where $\mathscr{D}^{\alpha}$ is the Caputo derivative with respect to time given by

$$
\mathscr{D}^{\alpha} \omega(t)=\frac{1}{\Gamma(n-v)} \int_{0}^{t}(t-\theta)^{m-v-1} \omega^{(k)}(\theta) d \theta, \quad n-1<\alpha \leq n, n \in \mathbb{N},
$$

where $S(t), I(t), P(t)$ are the densities of susceptible prey, infected prey, and predator populations, respectively. In this model, $r$ is the reproduction number of the prey population, and it is assumed that this infection cannot be transmitted vertically, in other words, mother-child, and the predator cannot be infected by this infection after a direct contact (predation) with infected prey. We use $e$ to denote the conversion rate of prey biomass (infected or susceptible) into predator biomass. Moreover, $\mu$ is the death rate of the prey population, and $m$ denotes the natural mortality of the predator population. The transmission rate of the prey population (infection rate) is described by $\delta$. The functional $(\lambda+a P) P S$ (resp. $(\lambda+a P) P I)$ is the hunting cooperation functional [22]. Our main motivation in this article is to study the influence of infection on the evolution of ecological species. Further, we study the influence of $\delta$ on the evolution of species. Moreover, the influence of the hunting cooperation functional on the spread of this infectious disease is studied. Accordingly, the general structure of this article is as follows. In the next section, we establish some useful tools for dealing with the fractional-order derivative operator. The equilibrium points of the system are calculated in Sect. 3. Section 4 is devoted to studying the stability of these equilibrium points. A numerical scheme is used in the fifth section for plotting the solution of model (1), where some graphical representations are used for verifying the expected mathematical results. Finally, the concluding section ends the paper.

\section{Equilibria of the model}

In this section, the equilibrium points of system (1) along with their epidemiological relevancies are investigated. The equilibria of system (1) are the solution to the following system:

$$
\left\{\begin{array}{l}
0=r(S+I)-(\lambda+a P) P S-\delta S I-\mu S \\
0=\delta S I-(\lambda+a P) P I-\mu I \\
0=e(\lambda+a P) P(S+I)-m P .
\end{array}\right.
$$

Obviously, $(S, I, P)=(0,0,0)$ is an equilibrium point of model (1) which models the extinction of the three species. Now we consider other particular cases.

Case 1 We seek equilibriums that have a zero density of predators (predator-free equilibriums), which are written as $E_{1}=\left(S_{1}, I_{1}, 0\right)$, which represents the extinction of the predator population. By replacing this result in the second equation of (2), we obtain 
$S_{1}=\frac{\mu}{\delta}$. By replacing these results in the first equation of (2), we get

$$
r S_{1}+r I_{1}-\delta S_{1} I_{1}-\mu S_{1}=0
$$

Hence,

$$
I_{1}=\frac{\delta S_{1}-r}{S_{1}(r-\mu)}
$$

which exists if the following assumption holds:

$$
\left(H_{1}\right) \quad\left(\delta S_{1}-r>0 \text { and } r>\mu\right) \text { or } \quad\left(\delta S_{1}-r<0 \text { and } r<\mu\right) \text {. }
$$

Case 2 We look for equilibrium without infection (infection-free equilibrium), which is elaborated by the presence of the susceptible prey and predator population only. This equilibrium is expressed as $E_{2}=\left(S_{2}, 0, P_{2}\right)$. Using the fact that $I_{2}=0$, system (2) becomes

$$
\left\{\begin{array}{l}
0=r S-(\lambda+a P) P S-\mu S, \\
0=e(\lambda+a P) P S-m P .
\end{array}\right.
$$

Multiplying the first equation of (3) by $e$ and adding it to the second equation, we obtain

$$
e(r-\mu) S_{2}-m P_{2}=0,
$$

hence,

$$
P_{2}=\frac{e}{m} S_{2}(r-\mu)
$$

with $r>\mu$. Replacing (4) in the second equation of system (3), we get

$$
\frac{a e^{2}}{m} S_{2}^{2}(r-\mu)+e \lambda S_{2}-m=0
$$

Obviously, Eq. (5) has a unique positive solution

$$
S_{2}=\frac{-e m \lambda+m \sqrt{e^{2} \lambda^{2}+4 a e^{2}(r-\mu)}}{2 a e^{2}} .
$$

The infection-free equilibrium exists if and only if the following condition holds:

$$
\left(H_{2}\right) \quad r>\mu .
$$

Case 3 In this case, we look for the positive equilibrium which models the existence of the three species. This equilibrium is indicated by $E_{*}=\left(S_{*}, I_{*}, P_{*}\right)$. Multiplying the 
first and second equations of system (2) by $e$, then adding them to the third equation, we obtain

$$
r\left(S_{*}+I_{*}\right)-\mu S_{*}-\mu I_{*}-m P_{*}=0 .
$$

So, it gives

$$
P_{*}=\frac{r-\mu}{m}\left(S_{*}+I_{*}\right)
$$

where $r>\mu$. Replacing (6) in the third equation of system (2), we obtain the following equation:

$$
\frac{e(r-\mu)}{m}\left(\lambda+\frac{a(r-\mu)}{m}\left(S_{*}+I_{*}\right)\right)-m=0 .
$$

By some straightforward calculations, we obtain

$$
S_{*}+I_{*}=\frac{m\left(m^{2}-\lambda(e r-\mu)\right)}{e a(r-\mu)^{2}} .
$$

Replacing (7) in (6), one gets

$$
P_{*}=\frac{m^{2}-\lambda(e r-\mu)}{e a(r-\mu)} .
$$

Replacing $P_{*}$ in the second equation of system (2), one achieves

$$
S_{*}=\frac{1}{\delta}\left(\lambda+a P_{*}\right) P_{*}+\mu
$$

Using the explicit expression of $S_{*}$ obtained in (9) along with (7) leads to

$$
I_{*}=\frac{m}{e a(r-\mu)^{2}}\left(m^{2}-\lambda(e r-\mu)\right)-S_{*} .
$$

For the epidemiological relevance of this equilibrium, we must have $S_{*}, I_{*}, P_{*}>0$. By taking a look at (8) and (9), we can guarantee that $r>\mu$ and $m^{2}+\lambda \mu>e r \lambda$ are necessary and sufficient conditions for having $S_{*}, P_{*}>0$. Now, we need an additional condition for guaranteeing the positivity of $I_{*}$.

In fact, using (6) we have

$$
m P_{*}=(r-\mu)\left(S_{*}+I_{*}\right)
$$

hence

$$
I_{*}=\frac{m P_{*}}{r-\mu}-\frac{P_{*}}{\delta}\left(\lambda+a P_{*}\right)+\mu,
$$

then $I_{*}>0$ if and only if

$$
\delta>\delta_{*}=\frac{m P_{*}+\mu(r-\mu)}{(r-\mu)\left(\lambda+a P_{*}\right) P_{*}} .
$$


Then we can summarize all the necessary existing conditions of the equilibrium point as follows:

$$
\left(H_{3}\right) \quad r>\mu, \quad m^{2}+\lambda \mu>e r \lambda \text { and } \delta>\delta_{*} \text {. }
$$

\section{The asymptotic behavior of equilibria}

Our main interest in this section is to determine the local stability of the equilibria calculated in the previous section. For that purpose we calculate the Jacobian matrix of system (1) given by

$$
J_{(S, I, P)}=\left(\begin{array}{ccc}
r-(\lambda+a P) P-\delta I-\mu & r-\delta S & -(\lambda+2 a P) S \\
\delta I & \delta S-(\lambda+a P) P-\mu & -(\lambda+2 a P) I \\
e(\lambda+a P) P & e(\lambda+a P) P & e(\lambda+2 a P)(S+I)-m
\end{array}\right)
$$

At the origin $E_{0}=(0,0,0)$, the Jacobian matrix (11) becomes

$$
J_{(0,0,0)}=\left(\begin{array}{ccc}
r-\mu & r & 0 \\
0 & -\mu & 0 \\
0 & 0 & -m
\end{array}\right)
$$

Obviously, the Jacobian matrix (12) has the following eigenvalues: $\vartheta_{1}=r-\mu<0, \vartheta_{2}=-\mu$, $\vartheta_{3}=-m$. Note that $\vartheta_{2}, \vartheta_{3}$ verify the condition $\left|\arg \left(\vartheta_{i}\right)\right|>\frac{\alpha \pi}{2}$. Hence the eigenvalue $\vartheta_{1}$ dominates the stability of the extinction equilibrium $E_{0}$, hence we deduce the following results.

Lemma 1 If $r<\mu$, then the equilibrium $E_{0}$ is locally stable and unstable if $r>\mu$.

On the other hand, for the equilibrium point $E_{1}=\left(S_{1}, I_{1}, 0\right)$, the Jacobian matrix (11) is calculated as

$$
J_{\left(S_{1}, I_{1}, 0\right)}=\left(\begin{array}{ccc}
r-\delta I_{1}-\mu & r-\delta S_{1} & -\lambda S_{1} \\
\delta I_{1} & 0 & -\lambda I_{1} \\
0 & 0 & e \lambda\left(S_{1}+I_{1}\right)-m
\end{array}\right)
$$

It is easy to see that $\vartheta_{3}=e \lambda\left(S_{1}+I_{1}\right)-m$ is an eigenvalue of the Jacobian matrix (13), hence the positivity of this quantity is expressed as

$$
\vartheta_{3}= \begin{cases}> & \text { if } \lambda>\lambda_{2}=\frac{m}{e\left(S_{1}+I_{1}\right)} \\ < & \text { if } \lambda<\lambda_{2}\end{cases}
$$

Note that under the condition $\lambda>\lambda_{2}$ we have the instability of the equilibrium $E_{1}$. Now, we presume that $\lambda<\lambda_{2}$, which means that $\vartheta_{3}<0$. This condition means that the other two eigenvalues of the Jacobian matrix (13) determine the stability (resp. instability) of the equilibrium $E_{1}$. The remaining eigenvalues are the eigenvalues of the following matrix:

$$
J_{1}=\left(\begin{array}{cc}
r-\delta I_{1}-\mu & -\lambda S_{1} \\
\delta I_{1} & 0
\end{array}\right) \text {. }
$$


Obviously, the trace and the determinant of matrix (14) are

$$
\begin{aligned}
& \operatorname{Tr}_{1}=r-\mu-\delta I_{1}, \\
& \operatorname{Det}_{1}=-\delta I_{1}(r-\mu)<0 .
\end{aligned}
$$

Using the fact that $\operatorname{Det}_{1}<1$, we can deduce that one of the eigenvalues of matrix (14) verifies $|\arg (\vartheta)|<\frac{\alpha \pi}{2}$. Hence, the equilibrium $E_{2}=\left(S_{2}, I_{2}, 0\right)$ is always unstable. We resume the obtained results in the following lemma.

Lemma 2 The equilibrium $E_{2}=\left(S_{2}, I_{2}, 0\right)$ is unstable whenever it exists.

Now, we focus on the stability of the infection-free equilibrium $\left(S_{2}, 0, P_{2}\right)$. At this equilibrium, the Jacobian matrix (11) becomes

$$
J_{\left(S_{2}, 0, P_{2}\right)}=\left(\begin{array}{ccc}
r-\left(\lambda+a P_{2}\right) P_{2}-\mu & r-\delta S_{2} & -\left(\lambda+2 a P_{2}\right) S_{2} \\
0 & \delta S_{2}-\left(\lambda+a P_{2}\right) P_{2}-\mu & 0 \\
e\left(\lambda+a P_{2}\right) P_{2} & e\left(\lambda+a P_{2}\right) P_{2} & e\left(\lambda+2 a P_{2}\right) S_{2}-m
\end{array}\right) .
$$

We remark that $\vartheta_{2}=\delta S_{2}-\left(\lambda+a P_{2}\right) P_{2}-\mu$, the positivity of $\vartheta_{2}$ is given by

$$
\left\{\begin{array}{l}
\vartheta>0 \quad \text { if and only if } \quad \delta>\delta_{2}=\frac{\left(\lambda+a P_{2}\right) P_{2}+\mu}{S_{2}} \\
\vartheta \leq 0 \quad \text { if and only if } \quad \delta \leq \delta_{2} .
\end{array}\right.
$$

The other two eigenvalues are the eigenvalues of the following matrix:

$$
J_{2}=\left(\begin{array}{cc}
0 & -\left(\lambda+2 a P_{2}\right) S_{2} \\
e\left(\lambda+a P_{2}\right) P_{2} & e a P_{2} S_{2}-m
\end{array}\right)
$$

The trace and the determinant of matrix (16) are

$$
\begin{aligned}
& \operatorname{Tr}_{2}=e a P_{2} S_{2}>0 \\
& \operatorname{Det}_{2}=e\left(\lambda+a P_{2}\right) P_{2} S_{2}\left(\lambda+2 a P_{2}\right)>0 .
\end{aligned}
$$

Note that in the case of the integer differential equation we deduce that this equilibrium is always unstable. But in the context of fractional differential calculus, this equilibrium point can be stable. In this regards, we presume that the eigenvalues of matrix (16) are written in the form $\vartheta=P+i Q$, which verifies

$$
\tan ^{2}[\arg (\vartheta)]=\frac{Q^{2}}{P^{2}}=\frac{4\left(\lambda+a P_{2}\right)\left(\lambda+2 a P_{2}\right)}{e a P_{2} S_{2}}-1
$$

Hence we can deduce that the eigenvalue $\vartheta$ verifies the condition $|\arg (\vartheta)|<\frac{\alpha \pi}{2}$ if the following condition holds:

$$
4\left(\lambda+a P_{2}\right)\left(\lambda+2 a P_{2}\right)>e a P_{2} S_{2}\left(1+\tan ^{2}\left(\frac{\alpha \pi}{2}\right)\right)
$$

Now we can resume the stability conditions of the equilibrium $E_{2}$ in the following lemma. 
Lemma 3 Assume that $r>\mu$, then the disease-free equilibrium $E_{2}$ is locally stable if

$$
\delta \leq \delta_{2} \quad \text { and } 4\left(\lambda+a P_{2}\right)\left(\lambda+2 a P_{2}\right)>e a P_{2} S_{2}\left(1+\tan ^{2}\left(\frac{\alpha \pi}{2}\right)\right)
$$

else it is unstable.

Now, let us study the stability of the interior equilibrium point. At this point, the Jacobin matrix is calculated as follows:

$$
J_{\left(S_{*}, I_{*}, P_{*}\right)}=\left(\begin{array}{ccc}
-\frac{r I^{*}}{S_{*}} & r-\delta S_{*} & -\left(\lambda+2 a P_{*}\right) S_{*} \\
\delta I_{*} & 0 & -\left(\lambda+2 a P_{*}\right) I_{*} \\
e\left(\lambda+a P_{*}\right) P_{*} & e\left(\lambda+a P_{*}\right) P_{*} & 0
\end{array}\right) .
$$

By solving the following equation, the eigenvalues of the Jacobi matrix (17) can be determined as follows:

$$
\Delta=\vartheta^{3}+\Psi_{2} \vartheta^{2}+\Psi_{1} \vartheta+\Psi_{0}
$$

where

$$
\begin{aligned}
& \Psi_{2}=\frac{r I_{*}}{S_{*}}, \\
& \Psi_{1}=e P_{*}\left(\delta S_{*}-\mu\right)\left(\lambda+a P_{*}\right)+\delta I_{*}\left(\delta S_{*}-\mu\right)+e S_{*} P_{*}\left(\lambda+a P_{*}\right)\left(\lambda+2 a P_{*}\right), \\
& \Psi_{0}=e P_{*} I_{*}\left(\lambda+a P_{*}\right)\left[\frac{r I_{*}}{S_{*}}\left(\delta S_{*}-\mu\right)-\left(\delta S_{*}-r\right)^{2}+\delta S_{*} I_{*}(\lambda+2 a P *)\right],
\end{aligned}
$$

we let

$$
G=18 \Psi_{2} \Psi_{1} \Psi_{0}+\left(\Psi_{2} \Psi_{1}\right)^{2}-4 \Psi_{0} \Psi_{2}^{3}-4 \Psi_{1}^{3}-27 \Psi_{0}^{3}
$$

Using the Routh-Hurwitz for fractional calculus, we arrive at the stability conditions of the positive equilibrium, which is resumed in the following theorem.

Theorem 1 We guarantee the stability of the positive equilibrium if one of the following assumptions holds:

(i) $G>0, \Psi_{2}>0, \Psi_{0}>0, \Psi_{2} \Psi_{1}>\Psi_{0}$.

(ii) $G<0, \Psi_{2} \geq 0, \Psi_{1} \geq 0, \Psi_{0} \geq 0$, and $\alpha<\frac{2}{3}$.

\section{A numerical scheme for system (1)}

We consider the following fractional model:

$$
\mathscr{D}^{\alpha} W(t)=H(t, W(t)) .
$$

Applying the fundamental theorem of fractional calculus on (1), we arrive at

$$
W(t)-W(0)=\frac{1}{\Gamma(\alpha)} \int_{0}^{t} H(\omega, W(\omega))(t-\omega)^{\alpha-1} d \omega .
$$


Putting $t=t_{n}=n \hbar$ in (19), yields

$$
W\left(t_{n}\right)=W(0)+\frac{1}{\Gamma(\alpha)} \sum_{i=0}^{n-1} \int_{t_{i}}^{t_{i+1}} H(\omega, W(\omega))\left(t_{n}-\omega\right)^{\alpha-1} d \omega
$$

Now, approximate $H(t, V(t))$ by

$$
\left.H(t, W(t)) \approx H\left(t_{i+1}, W_{i+1}\right)+\frac{t-t_{i+1}}{\hbar}\left(H\left(t_{i+1}, W_{i+1}\right)\right)-H\left(t_{i}, W_{i}\right)\right), \quad t \in\left[t_{i}, t_{i+1}\right],
$$

where $W_{i}=W\left(t_{i}\right)$.

Substituting (21) into (20) and following the procedure used in [11, 12], we get

$$
W_{n}=W_{0}+\hbar^{\alpha}\left(\Theta_{n} H\left(t_{0}, W_{0}\right)+\sum_{i=1}^{n} \Upsilon_{n-i} H\left(t_{i}, W_{i}\right)\right)
$$

where

$$
\begin{aligned}
& \Theta_{n}=\frac{(n-1)^{\alpha+1}-n^{\alpha}(n-\alpha-1)}{\Gamma(\alpha+2)}, \\
& \Upsilon_{n}= \begin{cases}\frac{1}{\Gamma(\alpha+2)}, & n=0, \\
\frac{(n-1)^{\alpha}-2 n^{\alpha}+(1+n)^{\alpha}}{\Gamma(\alpha+2)}, & n=1,2, \ldots .\end{cases}
\end{aligned}
$$

If we try to use the numerical method presented in formula (22) to solve problem (1), we obtain the following iterative schemes:

$$
\begin{aligned}
& S_{n}=S_{0}+\hbar^{\alpha}\left(\Theta_{n} H_{1}\left(S_{0}, I_{0}, P_{0}\right)+\sum_{i=1}^{n} \Upsilon_{n-i} H_{1}\left(S_{i}, I_{i}, P_{i}\right)\right), \\
& I_{n}=I_{0}+\hbar^{\alpha}\left(\Theta_{n} H_{2}\left(S_{0}, I_{0}, P_{0}\right)+\sum_{i=1}^{n} \Upsilon_{n-i} H_{2}\left(S_{i}, I_{i}, P_{i}\right)\right), \\
& P_{n}=P_{0}+\hbar^{\alpha}\left(\Theta_{n} H_{3}\left(S_{0}, I_{0}, P_{0}\right)+\sum_{i=1}^{n} \Upsilon_{n-i} H_{3}\left(S_{i}, I_{i}, P_{i}\right)\right),
\end{aligned}
$$

where

$$
\begin{aligned}
& H_{1}(S, I, P)=r(S+I)-(\lambda+a P) P S-\delta S I-\mu S, \\
& H_{2}(S, I, P)=\delta S I-(\lambda+a P) P I-\mu I, \\
& H_{3}(S, I, P)=e(\lambda+a P) P(S+I)-m P .
\end{aligned}
$$

\section{Graphical representations}

In this section, we verify the obtained mathematical results using several graphical representations. The plots are offered in the following manner.

Fig. 1 The stability of the infection-free equilibrium $E_{2}=(1,0,1)$ is clear in this figure. In fact, the used values verify the existence condition of this equilibrium $(r>\mu)$. The following values are considered for the values $r=1.5, \lambda=0.5, a=0.5, \delta=0.5, \mu=0.5$, $e=0.5, m=0.5, \alpha=0.9$ and the initial conditions $\left(S_{0}, I_{0}, P_{0}\right)=(0.2,0.7,0.6)$. 

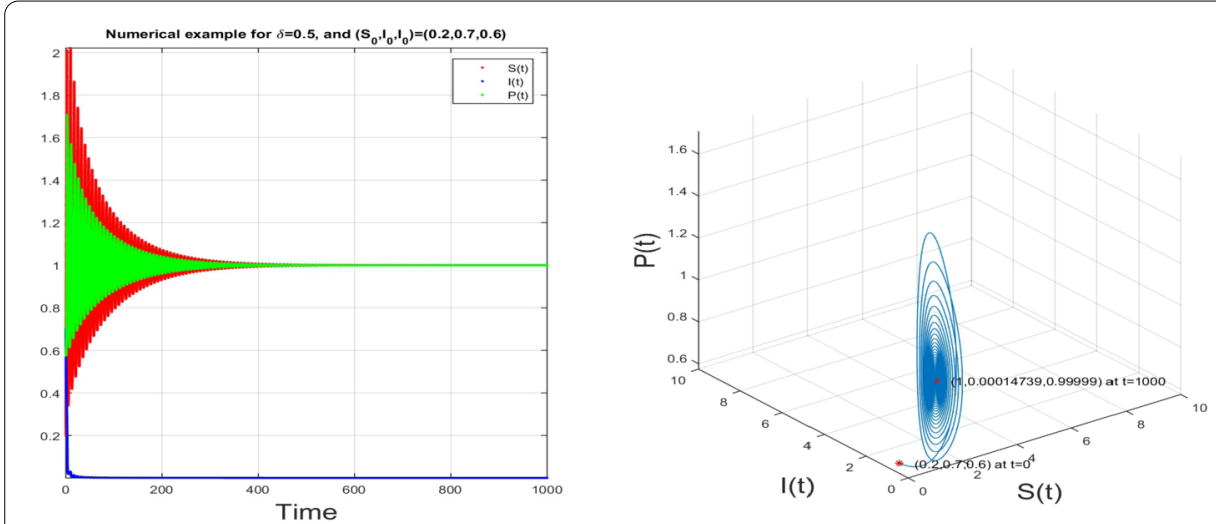

Figure 1 The local stability of infection-free equilibrium

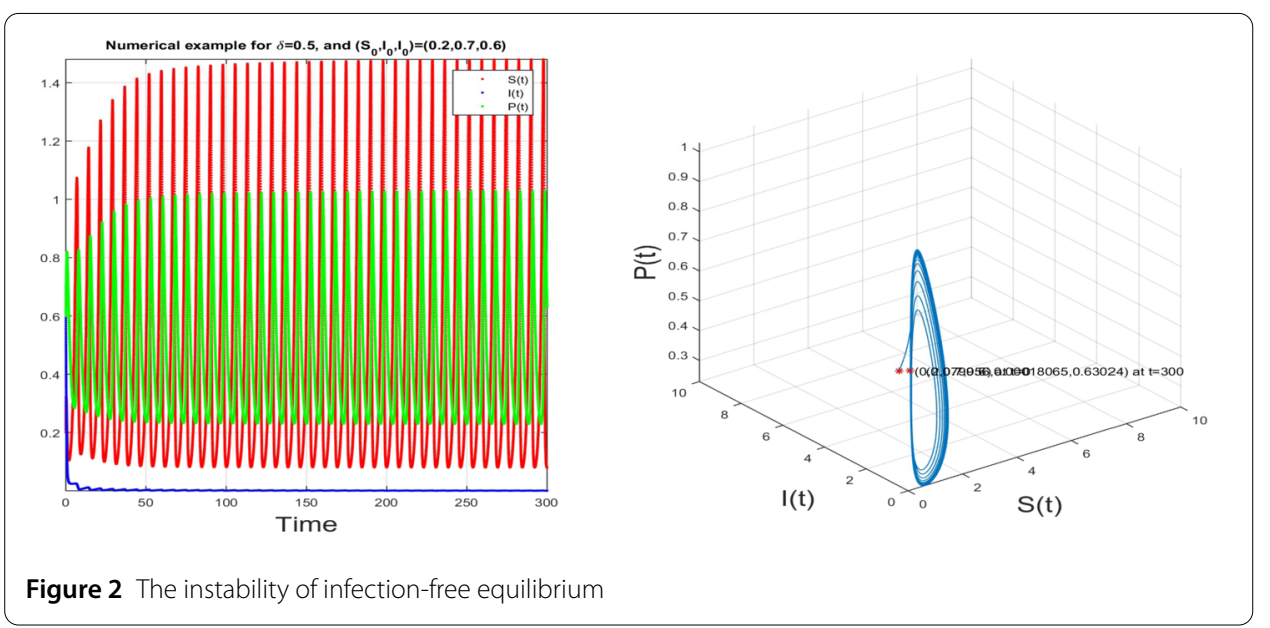

Fig. 2 In this figure we got the instability of the infection-free equilibrium. In fact, the used values verify the existence condition of this equilibrium $(r>\mu)$. The following values $r=1.5, \lambda=0.5, a=3.5, \delta=0.5, \mu=0.5, e=0.5, m=1.5, \alpha=0.9$ and the initial conditions $\left(S_{0}, I_{0}, P_{0}\right)=(0.2,0.7,0.6)$ are considered.

Fig. 3 In this figure we got the stability of the positive equilibrium. In fact, the used values verify the existence condition of this equilibrium $(r>\mu)$. The following values $r=3.5$, $\lambda=3.05, a=0.8, \delta=2.5, \mu=0.15, e=0.05, m=0.3, \alpha=0.9$ and the initial conditions $\left(S_{0}, I_{0}, P_{0}\right)=(0.2,0.7,0.6)$ are considered.

Fig. 4 This figure shows the existence of oscillations generated by the instability of the positive equilibrium. The following values $r=0.55, \lambda=0.5, a=3.5, \delta=0.5, \mu=0.5$, $e=0.5, m=1.5, \alpha=0.9$ and the initial conditions $\left(S_{0}, I_{0}, P_{0}\right)=(0.2,0.7,0.6)$ are considered.

Fig. 5 This figure also shows the existence of oscillations generated by the instability of the positive equilibrium. The following values $r=0.75, \lambda=0.5, a=3.5, \delta=0.5, \mu=$ $0.5, e=0.5, m=1.5, \alpha=0.9$ and the initial conditions $\left(S_{0}, I_{0}, P_{0}\right)=(0.2,0.7,0.6)$ are considered. 

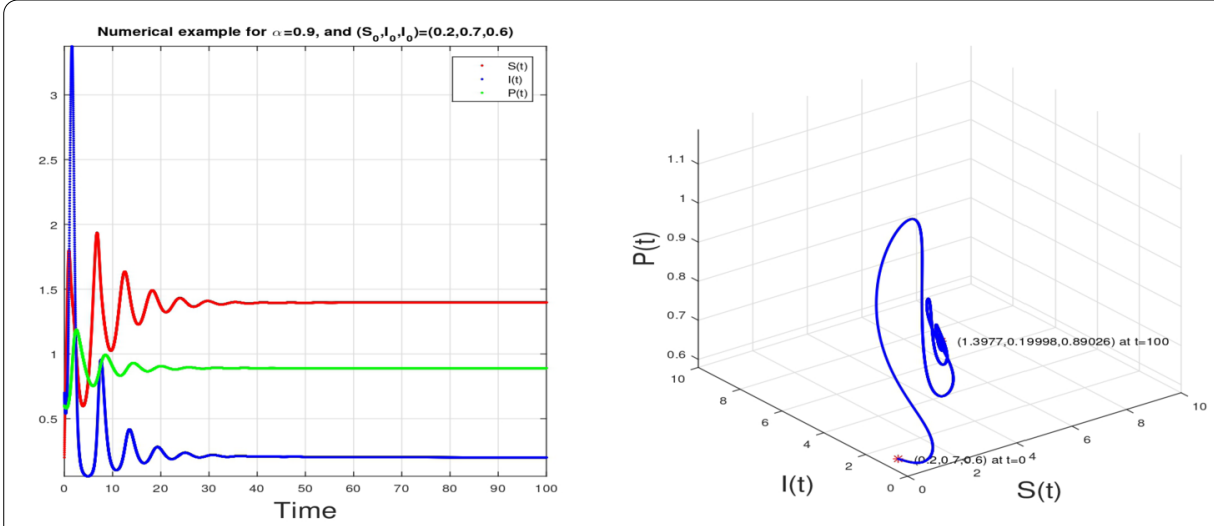

Figure 3 The instability of positive equilibrium
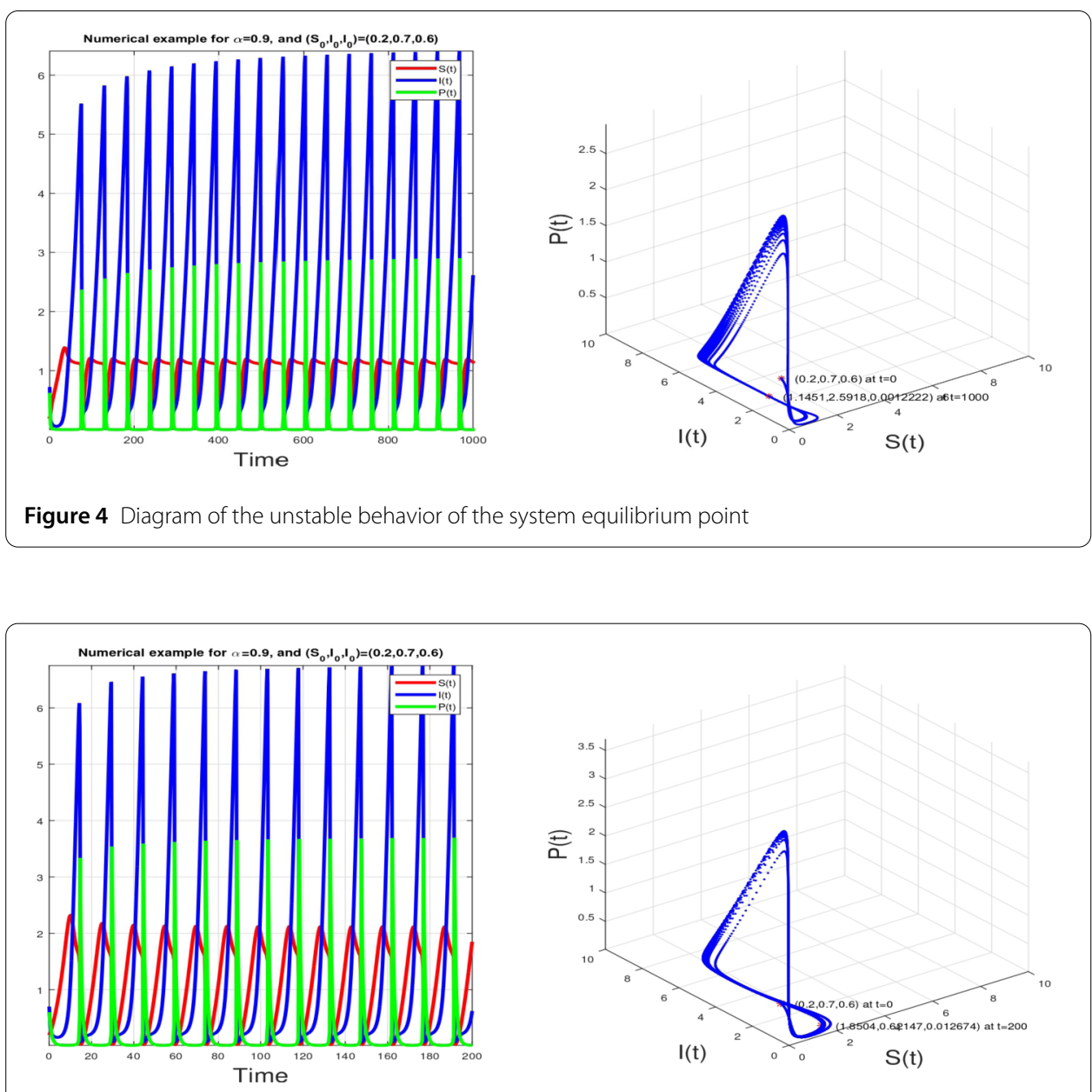

Figure 5 Diagram of the unstable behavior of the system equilibrium point

Fig. 6 This figure shows the existence of oscillations for the values $r=0.95, \lambda=0.5, a=$ $3.5, \delta=0.5, \mu=0.5, e=0.5, m=1.5, \alpha=0.9$, and the initial conditions $\left(S_{0}, I_{0}, P_{0}\right)=$ $(0.2,0.7,0.6)$ are considered. 

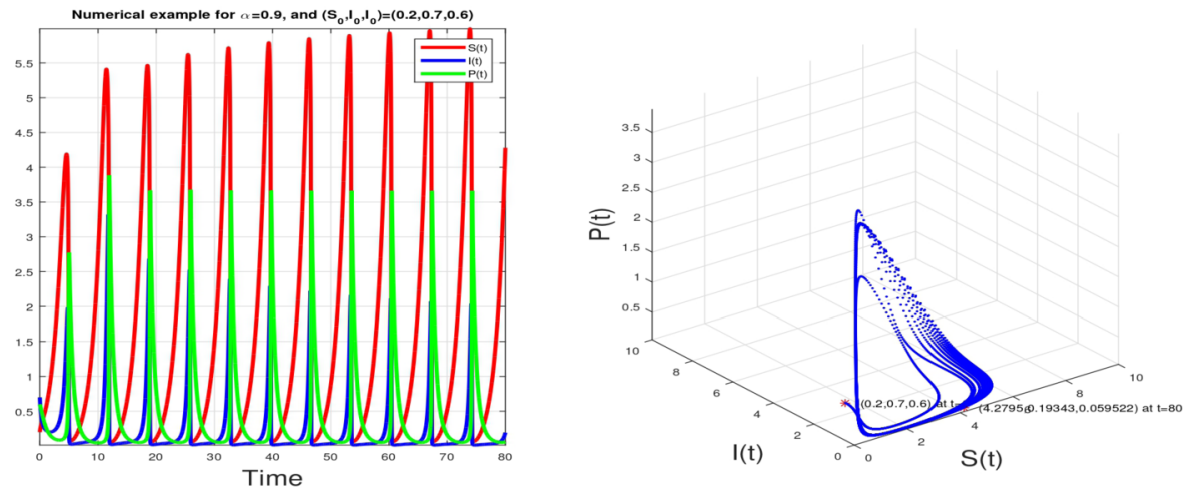

Figure 6 Diagram of the unstable behavior of the system equilibrium point
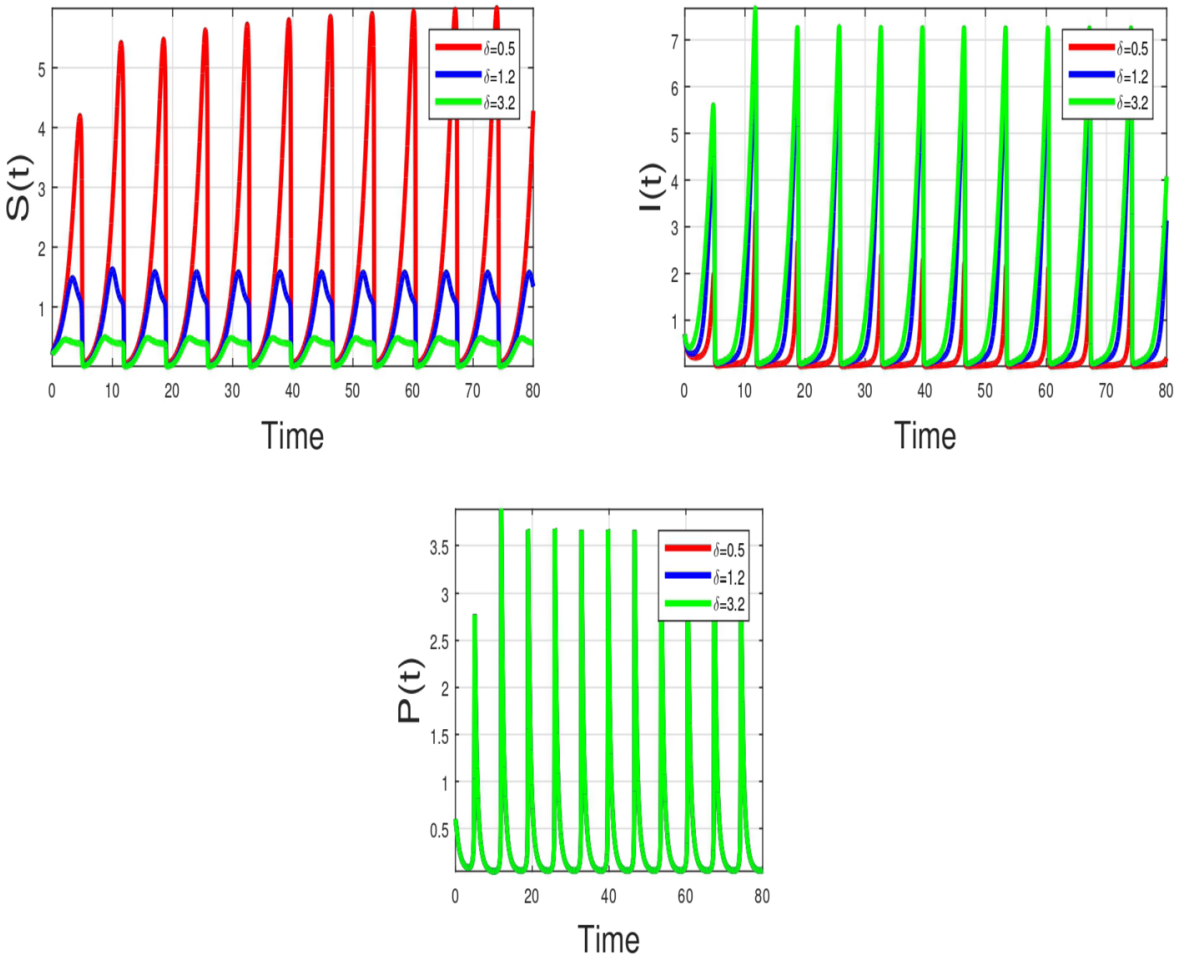

Figure 7 Influence of the infection rate $\delta$ on the evolution of the three species

Fig. 7 The influence of the infection rate on the evolution of the three species for the values $r=1.2, \lambda=0.5, a=3.5, \mu=0.5, e=0.5, m=1.5, \alpha=0.9$ and multi-values of $\delta$, and the initial conditions $\left(S_{0}, I_{0}, P_{0}\right)=(0.2,0.7,0.6)$ are considered.

Fig. 8 The influence of the memory rate $\alpha$ on the evolution of the three species for the values $r=3.5, \lambda=1.5, a=3.5, \delta=0.05, \mu=0.5, e=0.5, m=1.5$ and multi-values of $\alpha$, and the initial conditions $\left(S_{0}, I_{0}, P_{0}\right)=(0.2,0.7,0.6)$ are considered.

Fig. 9 The influence of the memory rate $\alpha$ on the evolution of the three species for the values $r=0.95, \lambda=0.5, a=3.5, \delta=0.5, \mu=0.5, e=0.5, m=1.5$ and multi-values of $\alpha$, and the initial conditions $\left(S_{0}, I_{0}, P_{0}\right)=(0.2,0.7,0.6)$ are considered. 

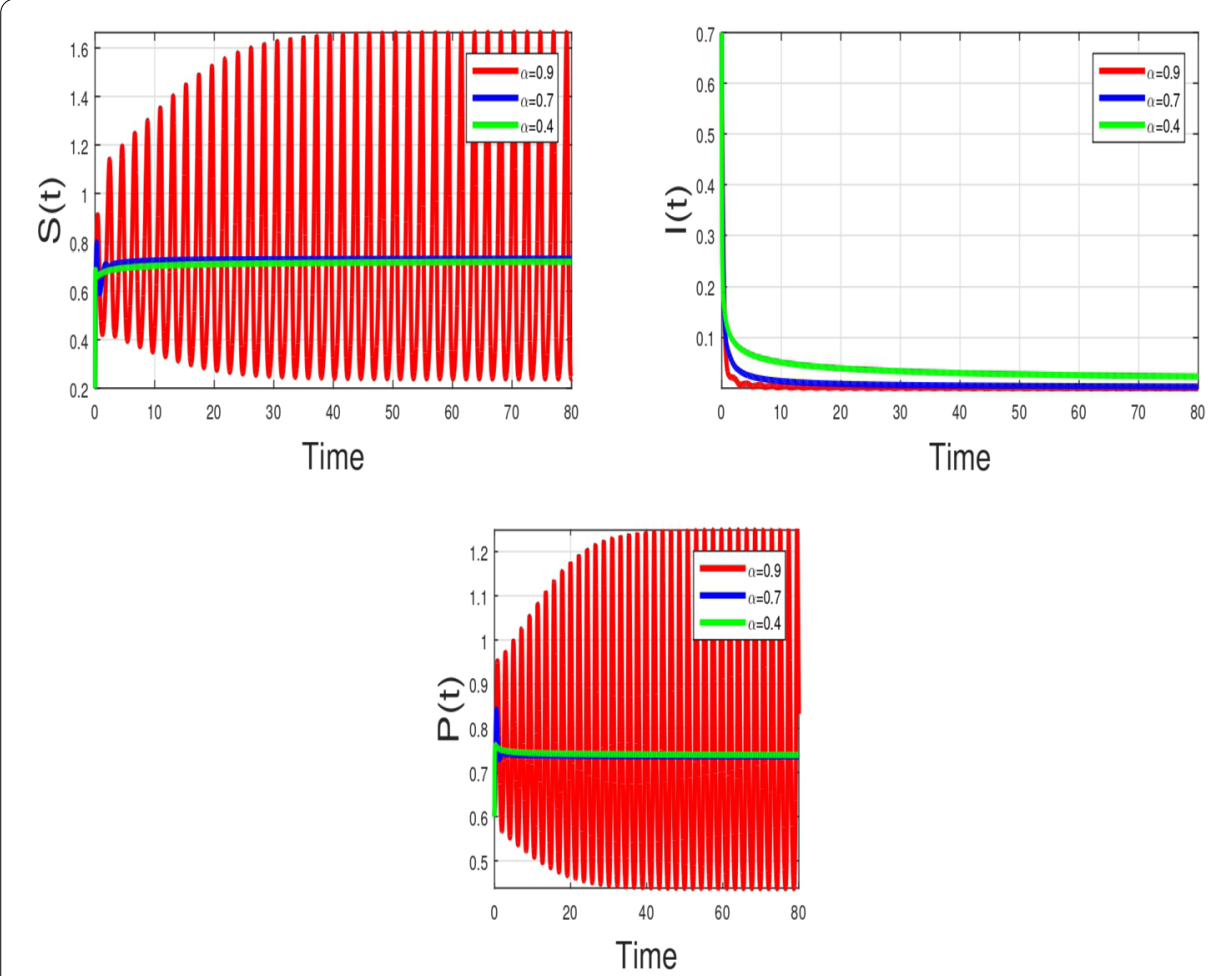

Figure 8 Influence of fractional-order derivative $\alpha$ on the evolution of the three species

\section{Conclusion}

In this paper, we dealt with the influence of infectious disease on the evolution of an ecological species using a fractional-order model. The fractional-order derivative stands for the memory which is responsible for the ecological fluctuation generated by the environment. It is assumed that this infection is fatal neither for the prey (after the infection) nor for the predator (after consuming an infected prey). Also, it is considered that the predator has a social behavior deduced by the cooperation in hunting the two prey (healthy and infected ones). Indeed, we obtained that system (1) can undergo many scenarios, as the extinction of the three populations which is deduced by the existence of the equilibrium $E_{0}$, the extinction of the predator population only without the infection. In this case, we have the persistence of the infection without the predator, which is deduced by the existence of the equilibrium $E_{1}$. This scenario cannot exist because of the instability of the equilibrium $E_{1}$, which is deducted from Lemma 3.2. Further, the extinction of the infection without predation is the most desired result, which represents the recovering of the ecological system from this infection. The stability of the infection-free equilibrium $E_{2}$ is the main result. We mention that in the case of the first-order derivative the equilibrium $E_{2}$ is always unstable because the eigenvalues of the Jacobian matrix verify $\operatorname{Tr}_{2}>0$ and $\operatorname{Det}_{2}>0$, but because of the expansion of the stability region generated by the time-fractional order derivative, we can have the stability of this equilibrium, which highlights the big influence of the memory on the ecological fluctuation. This result is supported numerically using graphical simulations plotted in Figs. 1 and 2. The existence of the three species is also possible where the stability of the equilibrium $E_{*}$ is possible. Figures $3,4,5,6$ confirm this result. 

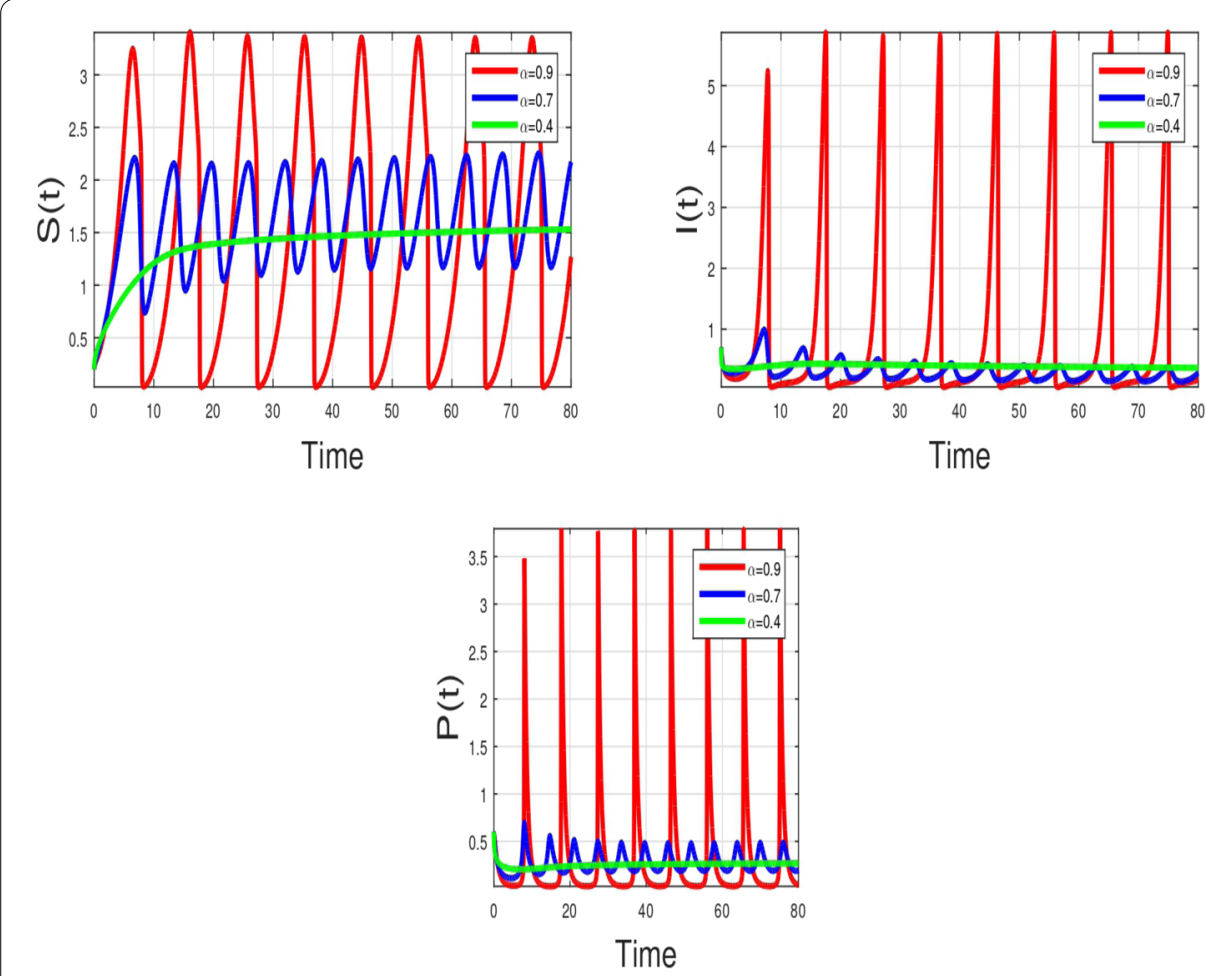

Figure 9 Influence of fractional-order derivative $\alpha$ on the evolution of the three species

Furthermore, this infection affects the final size of the prey (infected and susceptible) and has no influence on the predator temporal behavior. This feature can be clearly seen in Fig. 7. Also, the fractional-time derivative has a direct influence on the temporal behavior of the solution, as it is shown in Figs. 8 and 9. In these graphs, it is evident that the fractional derivative has a significant effect on the evolution of the existing species. This result signifies that under the same conditions the ecological species may not behave the same, and these figures (Fig. 8 and 9) confirm this presumption.

\section{Acknowledgements}

S. Djilali is partially supported by the DGRSTD of Algeria No.C00L03UN130120200004.

\section{Funding}

This research work is not supported by any funding agencies.

Availability of data and materials

Not applicable.

\section{Competing interests}

The authors declare that they have no competing interests.

\section{Authors' contributions}

All authors contributed equally and significantly in writing this paper. All authors have read and approved the final paper.

\section{Author details}

'Laboratoire d'Analyse Non Linéaire et Mathématiques Appliquées, Université de Tlemcen, Tlemcen, Algeria.

${ }^{2}$ Department of Mathematics, Faculty of Exact Sciences and Informatics, Hassiba Benbouali University, Chlef, Algeria.

${ }^{3}$ Department of Basic Science, Kermanshah University of Technology, Kermanshah, Iran. ${ }^{4}$ Department of Mathematics,

Faculty of Engineering and Natural Sciences, Bahçeşehir University, 34349 Istanbul, Turkey. 


\section{Publisher's Note}

Springer Nature remains neutral with regard to jurisdictional claims in published maps and institutional affiliations.

\section{Received: 2 November 2020 Accepted: 13 December 2020 Published online: 07 January 2021}

\section{References}

1. Akgül, A.: Analysis and new applications of fractal fractional differential equations with power law kernel. Discrete Contin. Dyn. Syst., Ser. S 0 (2018)

2. Akgül, A.: A novel method for a fractional derivative with non-local and non-singular kernel. Chaos Solitons Fractals 114, 478-482 (2018)

3. Araz, S.I.: Analysis of a Covid-19 model: optimal control, stability and simulations. Alex. Eng. J. (2020)

4. Atangana, A., Akgül, A.: Can transfer function and Bode diagram be obtained from Sumudu transform. Alex. Eng. J. (2020). https://doi.org/10.1016/j.aej.2019.12.02

5. Atangana, A., AkgulKolade, A., Owolabi, M.: Analysis of fractal fractional differential equations. Alex. Eng. J. 59, $117-1134(2020)$

6. Atangana, A., Araz, S.I.: Mathematical model of COVID-19 spread in Turkey and South Africa: Theory Methods Appl. medRxiv (2020)

7. Bentout, S., Tridane, A., Djilali, S., Touaoula, T.M.: Age-structured Modeling of COVID-19 Epidemic in the USA, UAE and Algeria, Alex. Eng. J. (2020). https://doi.org/10.1016/j.aej.2020.08.053

8. Capone, F., Carfora, M.F., De Luca, R., Torcicollo, I.: Turing patterns in a reaction-diffusion system modeling hunting cooperation. Math. Comput. Simul. 165, 172-180 (2019)

9. Chattopadhyay, J., Arino, O.: A predator-prey model with disease in the prey. Nonlinear Anal. 36, 747-766 (1999)

10. Cosner, C., DeAngelis, D., Ault, J., Olson, D.: Effects of spatial grouping on the functional response of predators. Theor. Popul. Biol. 56(1), 65-75 (1999)

11. Diethelm, K:: Smoothness properties of solutions of Caputo-type fractional differential equations. Fract. Calc. Appl. Anal. 10, 151-160 (2007)

12. Diethelm, K., Ford, N.J., Freed, A.D.: Detailed error analysis for a fractional Adams method. Numer. Algorithms 36, 31-52 (2004)

13. Djilali, S.: Herd behavior in a predator-prey model with spatial diffusion bifurcation analysis and Turing instability. J. Appl. Math. Comput. 58(1-2), 125-149 (2017)

14. Djilali, S.: Impact of prey herd shape on the predator-prey interaction. Chaos Solitons Fractals 120, 139-148 (2019)

15. Djilali, S.: Effect of herd shape in a diffusive predator-prey model with time delay. J. Appl. Anal. Comput. 9(2), 638-654 (2019)

16. Djilali, S.: Pattern formation of a diffusive predator-prey model with herd behavior and nonlocal prey competition. Math. Methods Appl. Sci. 43(5), 2233-2250 (2020)

17. Djilali, S:: Spatiotemporal patterns induced by cross-diffusion in predator-prey model with prey herd shape effect. Int. J. Biomath. 13(4), 2050030 (2020) https //doi.org/10.1142/S1793524520500308

18. Djilali, S., Bentout, S.: Spatiotemporal patterns in a diffusive predator-prey model with prey social behavior. Acta Appl. Math. 169, 125-143 (2020)

19. Djilali, S., Ghanbari, B.: Coronavirus pandemic a predictive analysis of the peak outbreak epidemic in South Africa, Turkey, and Brazil. Chaos Solitons Fractals 138, 109971 (2020)

20. Djilali, S., Ghanbari, B., Bentout, S., Mezouaghi, A.: Turing-Hopf bifurcation in a diffusive Mussel-Algae model with time-fractional-order derivative. Chaos Solitons Fractals 138, 109954 (2020)

21. Djilali, S., Touaoula, T.M., Miri, S.E.H.: A heroin epidemic model very general nonlinear incidence, treat-age, and global stability. Acta Appl. Math. 152(1), 171-194 (2017)

22. Duarte, J., Januario, C., Martins, N., Sardanyes, J.: Chaos and crises in a model for cooperative hunting a symbolic dynamics approach. Chaos 19(4), 043102 (2009)

23. Ghanabri, B., Djilali, S.: Mathematical and numerical analysis of a three-species predator-prey model with herd behavior and time fractional-order derivative. Math. Methods Appl. Sci. 43(4), 1736-1752 (2020)

24. Ghanabri, B., Djilali, S.: Mathematical analysis of a fractional-order predator-prey model with prey social behavior and infection developed in predator population. Chaos Solitons Fractals 138, 109960 (2020)

25. Hadeler, K.P., Freedman, H.I.: Predator-prey populations with parasitic infection. J. Math. Biol. 27, 609-631 (1989)

26. Han, L., Ma, Z., Hethcote, H.W.: Four predator prey models with infectious diseases. Math. Comput. Model. 34(7-8), 849-858 (2001)

27. Lotka, A.J.: Elements of Physical Ecology. Williams and Wilkins, New York (1925)

28. Owolabi, K.M., Atangana, A., Akgül, A.: Modelling and analysis of fractal-fractional partial differential equations: application to reaction—diffusion model. Alex. Eng. J. (2020). https://doi.org/10.1016/j.aej.2020.03.022

29. Pal, S., Pal, N., Samanta, S., Chattopadhyay, J.: Effect of hunting cooperation and fear in a predator-prey model. Ecol. Complex. 39, 100770 (2019)

30. Ryu, K., Ko, W.: Asymptotic behavior of positive solutions to a predator-prey elliptic system with strong hunting cooperation in predators. Physica A 531, 121726 (2019)

31. Sen, D., Ghorai, S., Banerjee, S.M.: Allee effect in prey versus hunting cooperation on predator - enhancement of stable coexistence. Int. J. Bifurc. Chaos 29(6), 1950081 (2019)

32. Singh, T., Dubey, R., Mishra, V.N.: Spatial dynamics of predator-prey system with hunting cooperation in predators and type I functional response. AIMS Math. 5, 673-684 (2020)

33. Song, D., Song, Y., Li, C.: Stability and turing patterns in a predator-prey model with hunting cooperation and Allee effect in prey population. Int. J. Bifurc. Chaos 30(09), 2050137 (2020)

34. Souna, F., Lakmeche, A., Djilali, S.: The effect of the defensive strategy taken by the prey on predator-prey interaction. J. Appl. Math. Comput. 64, 665-690 (2020)

35. Souna, F., Lakmeche, A., Djilali, S.: Spatiotemporal patterns in a diffusive predator-prey model with protection zone and predator harvesting. Chaos Solitons Fractals 140, 110180 (2020)

36. Souna, F., Lakmesh, A., Djilali, S.: The effect of the defensive strategy taken by the prey on predator-prey interaction. J. Appl. Math. Comput. (2020). https://doi.org/10.1007/s12190-020-01373-0 
37. Volterra, V:: Sui tentativi di applicazione della matematiche alle scienze biologiche e sociali. G. Econ. 23,436-458 (1901)

38. Wu, D., Zhao, M.: Qualitative analysis for a diffusive predator-prey model with hunting cooperative. Physica A 515 299-309 (2019)

39. Yan, S., Jia, D., Zhang, T., Yuan, S.: Pattern dynamics in a diffusive predator-prey model with hunting cooperations. Chaos Solitons Fractals 130, 109428 (2020)

40. Yavuz, M., Sene, N.: Stability analysis and numerical computation of the fractional predator-prey model with the harvesting rate. Fractal Fract. 4(3), 35 (2020)

41. Zhou, X., Cui, J., Shi, X., et al.: A modified Leslie-Gower predator-prey model with prey infection. J. Appl. Math. Comput. 33, 471-487 (2010)

Submit your manuscript to a SpringerOpen ${ }^{\circ}$ journal and benefit from:

- Convenient online submission

- Rigorous peer review

- Open access: articles freely available online

- High visibility within the field

- Retaining the copyright to your article

Submit your next manuscript at $\gg$ springeropen.com 